\title{
Das Seerosengleichnis
}

$\mathrm{V}$

or Kurzem poppte die Mail meines Freundes Helmut aus dem Silicon Valley auf. In der businessweek vom 21. Mai sei ein Artikel über den Stand der Künstlichen Intelligenz, den ich unbedingt lesen müsse. Darin ein Porträt über einen bedeutenden Paten der KI, Jürgen Schmidhuber.

Sofort fange ich an nachzulesen und lerne, dass Schmidhuber aus München stammt und auch hier studiert hat. Bereits mit 15 Jahren hat er die Vision, eine künstliche Intelligenz zu entwickeln, die den Menschen überlegen ist. Als Erwachsener arbeiten er und sein Team unermüdlich daran, das wahr zu machen. Heute nutzen Konzerne wie Google, Facebook und Amazon künstliche Intelligenz und generieren damit riesige Technik- und Marketing-Vorsprünge. Schmidhubers herausragende Entwicklung: das rückgekoppelte, neuronale Netzwerk, kurz RNN.

Ich stelle mir das System vor wie ein rotes Seil, das sich seinen Weg durch ein Labyrinth sucht. Ständig schlängelt es in Sackgassen, lernt daraus den Misserfolg und zieht sich wieder zurück zur letzten Kreuzung. Probiert einen neuen Weg, der funktioniert. Begreift das als Erfolg, merkt sich alles und wird immer klüger.

Auf dieser Basis kreiert Schmidhuber einen Weltbestseller: das Long-Short-Term-Memory Netzwerk (LSTM). Ein Super-RNN, das z.B. mit exzellenten Übersetzungstexten aus verschiedenen Sprachen trainiert werden und damit bald der beste Übersetzer der Welt werden kann. Das alles fasziniert mich und für mich steht fest: Ich muss Schmidhuber kennenlernen.

Wenige Wochen später sitze ich ihm im Frühstücksraum eines gutbürgerlichen Hotels im Münchner Süden gegenüber Er trägt weiß, kommt aber ohne seine legendäre Schiebermütze. Freundlich ist er, und nimmt sich Zeit. Wir sprechen darüber, dass die moderne Chip-Technik jetzt erstmals in der Menschheitsgeschichte so weit ist, mithilfe von LSTM die Welt zu verändern. Ich frage ihn, ob ihm das nicht zu lange gedauert habe. Er lacht auf.

„Nein. Ich hatte immer Freude daran. Ich wusste ja, dass das eines Tages sichtbar wird. Es ist wie in einem Teich, in dem eine Seerose wächst. Jeden Tag verdoppelt sie ihr Volumen. Erst eine Blüte, dann zwei, dann vier und so weiter. Lange Zeit sieht man sie nicht, doch dann ist die Wasseroberfläche plötzlich voller Seerosen.“

Da ist sie, die exponentielle Entwicklung der Künstlichen Intelligenz, die in den streng steigenden, monotonen Modus wechselt. Eine Rakete auf dem direkten Weg zum Mars.

Und jetzt? Gerade hat das kleine Kölner Start-up Deepl mit schlauer KI den Megaplayer Google Translate überholt. Und die chronisch unflexible Siri von Apple hat nun den KI-Spezialisten von Google Translate an Bord. München ist mit Googles Anzeigen ihres KI-Assistenten plakatiert. Es herrscht Aufbruchstimmung.

Jedes Internetunternehmen, jeder Händler und Maschinenbauer wird blitzschnell auf die 5-zeiligen LSTM Codes aufschalten müssen. Die Technik wird menschenfreundlicher, klüger und schneller werden. Die LSTMs sind patentfrei. Es gibt sie kostenlos in Programmbibliotheken mit allen wichtigen Tutorials.

Es ist, wie er es sich als 15-Jähriger erträumt hat. Jürgen Schmidhubers Entwicklungen führen zu einer aktiven, kreativen, emotionalen und humanoiden KI. Oder wie er ês in seinem Satz in dem businessweek Artikel zusammenfasst: „Mein Team plant, den Lauf der Menschheitsgeschichte zu ändern“. Bleibt zu hoffen, dass wir in Deutschland auch schon bereit sind, daraus etwas für die Menschheit zu machèn.

Uwe Walter ist Storytelling- und Change-Experte für Medien- und Industrieunternehmen. Er berät so unterschiedliche Kunden wie YouTube-Stars, Start-ups, Blogger, Verlage, Radio- und Fernsehsender sowie Filmproduktionen. Seine Expertise: Wie generiere ich Reichweite durch zukunftssicheres Erzählen? 